\title{
Wearable Lumbar-Motion Monitoring Device with Stretchable Strain Sensors
}

\author{
Hiroyuki Nakamoto $\left(D^{1},{ }^{1}\right.$ Tokiya Yamaji, ${ }^{1}$ Akio Yamamoto, ${ }^{2}$ Hideo Ootaka, ${ }^{3}$ Yusuke Bessho, ${ }^{3}$ \\ Futoshi Kobayashi ${ }^{\circ},{ }^{1}$ and Rei Ono ${ }^{2}$ \\ ${ }^{1}$ Graduate School of System Informatics, Kobe University, 1-1 Rokkodai-cho, Nada, Kobe 657-8501, Japan \\ ${ }^{2}$ Graduate School of Health Science, Kobe University, 7-10-2 Tomogaoka, Suma, Kobe 654-0142, Japan \\ ${ }^{3}$ Bando Chemical Industries Ltd, 4-6-6 Minatojima Minamimachi, Chuo, Kobe 650-0047, Japan \\ Correspondence should be addressed to Hiroyuki Nakamoto; nakamoto@panda.kobe-u.ac.jp
}

Received 5 June 2018; Accepted 3 September 2018; Published 8 October 2018

Guest Editor: Aftab M. Hussain

Copyright (c) 2018 Hiroyuki Nakamoto et al. This is an open access article distributed under the Creative Commons Attribution License, which permits unrestricted use, distribution, and reproduction in any medium, provided the original work is properly cited.

Low-back pain is a common affliction. Epidemiological analyses have reported that periodic cycles of lumbar flexion and rotation are major risk factors for low-back pain. To prevent low-back pain, a lumbar-motion monitoring device could help diagnosticians assess patients' risk for low-back pain. This study proposes such a device that uses lightweight stretchable strain sensors. Six of these strain sensors form a parallel-sensor mechanism that measures rotation angles of lumbar motion in three axes. The parallel-sensor mechanism calculates rotation angles from the lengths of the strain sensors iteratively. Experimental results reveal that the prototype device is effective for lumbar-motion measurement and significantly improves in terms of wearability over comparable devices.

\section{Introduction}

Low-back pain (LBP) is a common affliction in contemporary life. Globally, two-thirds of adults experience LBP to the extent that treatment is required $[1,2]$. Hoy et al. reported that the point prevalence of LBP is as much as $10 \%$, and the 1 -month prevalence is over $20 \%$ [3]. LBP sufferers often have difficulty maintaining healthy living habits and steady work.

Although the prevalence of LBP is not limited to a particular job category, industrial workers, nurses, and healthcare workers tend to experience a relatively high prevalence of LBP [4-11]. These workers frequently use their back muscles and spines in lumbar motions of flexion, rotation, and side bending. With epidemiological analysis, Hoogendoorn et al. revealed that periodic lumbar flexion and rotation is a major risk factor for LBP [12]. Overall, LBP risk is assessed in terms of accumulated lumbar motions and loads, and patients can benefit from alerts of excess lumbar motions to prevent the onset or worsening of LBP. Waters et al. estimated the risk for LBP in manual lifting tasks with calculations [13]. Norman et al. assessed the risk for LBP in automotive industries using peak motion and cumulative lumbar motions as risk factors [14]. These methods are difficult to compare with each other, as they use different metrics for understanding lumbar risk. A measurement system that can continuously record time-series data during lumbar motions of flexion, rotation, and side bending could provide comparable lumbar data for a range of industries and subject groups.

To obtain time-series measurements of the pressure between lumbar bones, Lisi et al. obtained in vivo measurements with a load sensor inserted between lumbar vertebrae [15]. Wilke et al. measured the pressure between lumbar vertebrae during various motions such as lying, sitting, walking, and lifting and verified the results of Nachemson's study using EMG signals [16, 17]. Although these sensors directly provide the loads on lumbar vertebrae for various motions, such an invasive procedure cannot be used to continuously monitor movements for workers in practical environments. For less-invasive applications, camera-based 
motion-capture systems are generally used to record human motions. Motion-capture systems require expensive cameras and markers on the bodies to be measured, and the measured subjects must remain within a small area. Wearable sensors could dramatically reduce costs and allow workers to move unimpeded while monitoring their movements.

Milea measured joint motion with lightweight and inexpensive bend sensors [18]. Though the materials used were simple and inexpensive, the three-axis motions possible at the lumbar joints cannot be measured with bend sensors alone.

Inertial measurement unit (IMU) systems have also been used to measure body motions. Since IMUs can estimate three-axis angles using accelerometers and gyroscopes, the measured values will include errors [19]. Marras et al. developed a lumbar-motion monitoring device that uses a threeaxis electrogoniometer [20]. With this apparatus, Marras et al. monitored lumbar motion for industrial workers [21] and health care workers [22]. This lumbar-motion monitor effectively recorded time-series data about lumbar motions. However, the device covered the subjects' low back and may have been heavy for the subjects to work with over the course of a full shift. The weight of such a device must be reduced significantly if workers' low backs are to be monitored continuously.

We therefore propose a novel lightweight lumbar-motion measurement device (LMMD). The key component of the device is a flexible and stretchable strain sensor. This strain sensor is lightweight and features low elasticity, high durability, and good repeatability [23]. The strain sensor we use has been applied to measurements of wrist and elbow motions [24] and rapid prototyping human interfaces [25]. These joints rotate in one or two axes and can be modeled with a simple equation. Since lumbar motion includes flexion, rotation, and side bending, lumbar motion must be modeled with three degrees of freedom. To solve this problem, we designed a parallel-sensor device with six strain sensors. This device is lighter than comparable devices, and tests reported in this paper show that it can effectively measure lumbar motion over time.

\section{Methods}

The stretchable strain sensor we chose is flexible and thin. The main material of the strain sensor is urethane elastomer. Three elastomer sheets sandwich two carbon-nanotube membranes. These carbon-nanotube membranes work as electrodes in the strain sensor and are flexible enough to expand or contract along with the elastomer sheets. The sandwiched carbon-nanotube membranes are effectively a parallel-plate capacitor, the capacitance of which changes as the strain sensor expands and contracts. Hence, the strain sensor works as a variable capacitor, accurate to within $5 \%$ of its range [23]. The elastomer sheets are $70 \mathrm{~mm}$ long and $15 \mathrm{~mm}$ wide. The electrodes are $50 \mathrm{~mm}$ long and $10 \mathrm{~mm}$ wide. The whole strain sensor is approx. $150 \mu \mathrm{m}$ thick [23]. The strain sensors we used have protective fabric on both sides, as shown in Figure 1. Snap buttons on both terminals are used to anchor terminals of the strain sensor. The strain

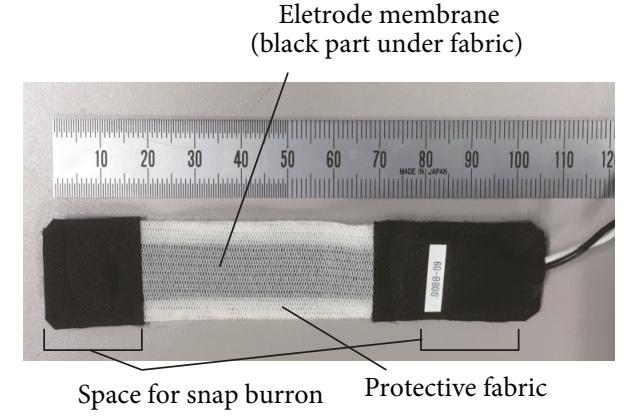

FIgURE 1: Stretchable strain sensor with protective fabric and snap buttons.

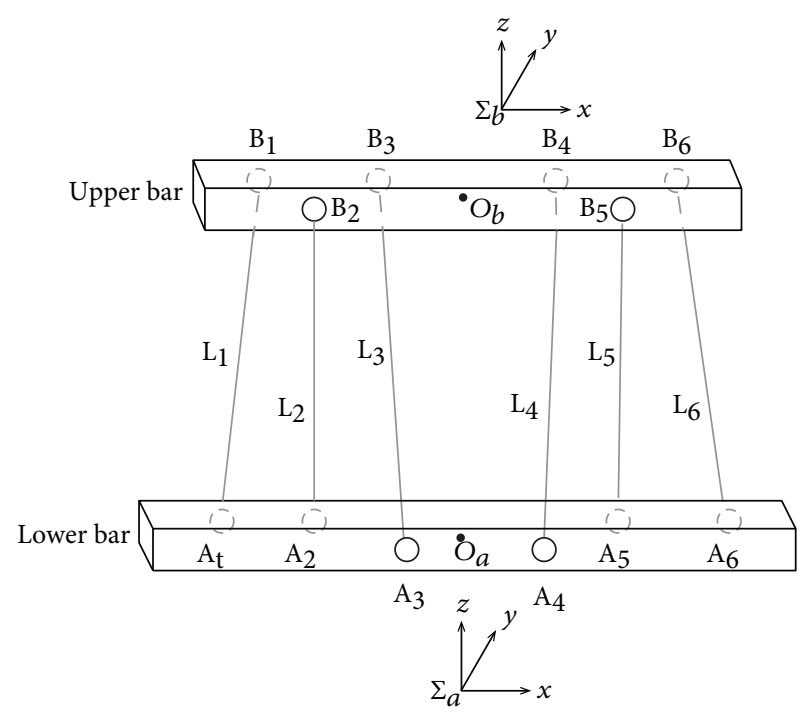

Figure 2: Model of the parallel-sensor mechanism. The origins of the coordinate systems of the lower and upper bars, $\Sigma_{a}$ and $\Sigma_{b}$, are coincident with the center points of the bars, $O_{a}$ and $O_{b}$, respectively.

sensor shown in Figure 1 is $110 \mathrm{~mm}$ long and $20 \mathrm{~mm}$ wide and weighs $4.1 \mathrm{~g}$.

The proposed measurement device is built around a parallel-sensor mechanism. The parallel-sensor mechanism is based on the Stewart platform [26]. A diagram of the parallel-sensor mechanism is shown in Figure 2. Two bars are connected by six links. The terminals of each link are attached to the lower and upper bars. When the links are actuators, the mechanism is a positional control device with six degrees of freedom [27]. If the links are linear encoders, the mechanism can function as a measurement system [28]. In this study, as in our previous work, the strain sensors serve as links and the device measures their lengths. When the upper bar moves or tilts relative to the lower bar, the strain sensors expand or contract. In that case, the relative threedimensional position and rotation of the upper bar can be calculated based on the lengths of the strain sensor.

The terminals of the links in the parallel-sensor model are labeled from $A_{1}$ to $A_{6}$ and from $B_{1}$ to $B_{6}$. The coordinate systems of the lower bar and upper bar are $\Sigma_{a}$ and $\Sigma_{b}$, 


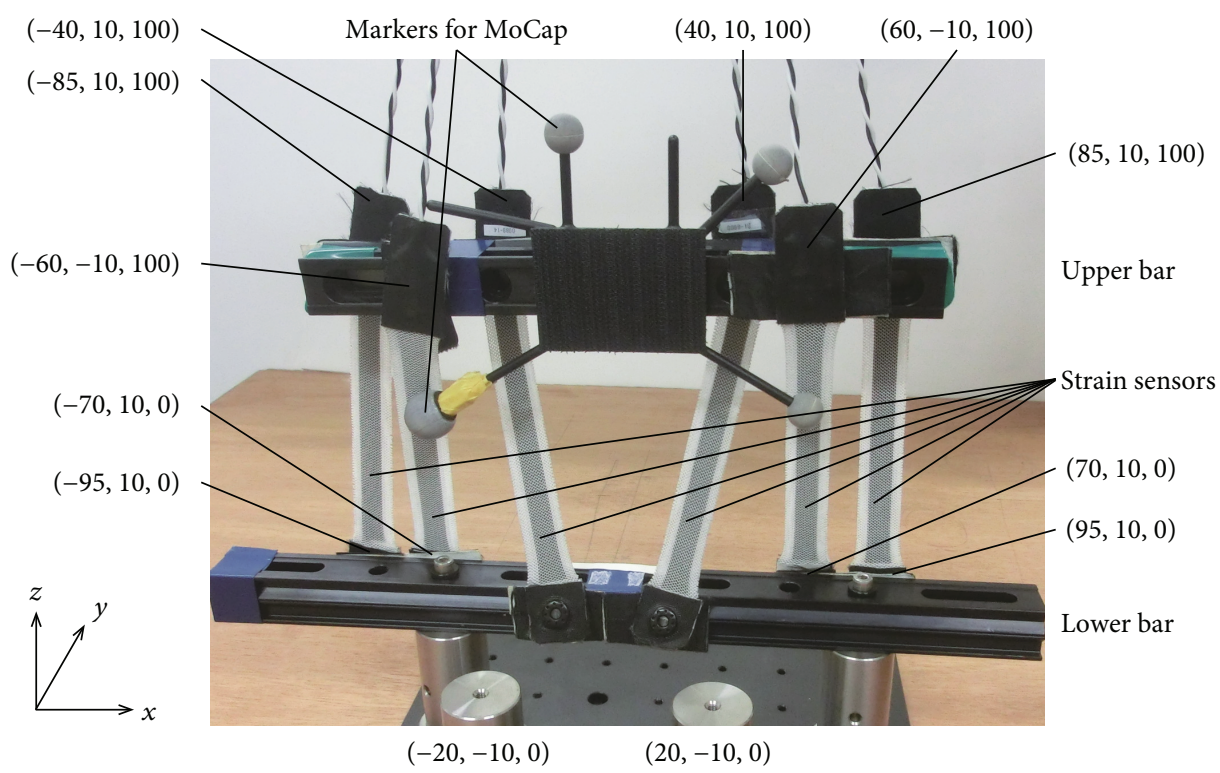

FIGURE 3: Parallel-sensor mechanism prototype. The origin of the coordinate system of the lower bar is at the center of the bar. The position of each terminal of the strain sensor in terms of the coordinate system is marked in units of $\mathrm{mm}$.

respectively. The origin $O_{b}$ of $\Sigma_{b}$ is represented as ${ }^{a} O_{b}=$ $\left[{ }^{a} \mathrm{O}_{b x}{ }^{a} \mathrm{O}_{b y}{ }^{a} \mathrm{O}_{b z}\right]^{T}$ in the coordinate system $\Sigma_{a}$. In coordination system $\Sigma_{a}$, the rotation angles of the upper bar around the $x$, $\mathrm{y}$, and $\mathrm{z}$ axes are represented as $\theta_{x}, \theta_{y}$, and $\theta_{z}$, respectively. The position and rotation of the origin of the upper bar are written as $P=\left[\begin{array}{llllll}{ }^{a} O_{b x} & { }^{a} O_{b y} & { }^{a} O_{b z} & \theta_{x} & \theta_{y} & \theta_{z}\end{array}\right]^{T}$. Coordinates from $\mathrm{B}_{1}$ to $\mathrm{B}_{6}$ in $\Sigma_{b}$ are represented as ${ }^{b} B_{i}=$ $\left[\begin{array}{lll}{ }^{b} B_{i x} & { }^{b} B_{i y} & { }^{b} B_{i z}\end{array}\right]^{T}$, where $i$ is the index number of the sensor and ranges from 1 to 6 . Similarly, the coordinates from $B_{1}$ to $\mathrm{B}_{6}$ in $\Sigma_{a}$ are represented as ${ }^{a} B_{i}=\left[\begin{array}{lll}{ }^{a} B_{i x} & { }^{a} B_{i y} & { }^{a} B_{i z}\end{array}\right]^{T}$, which is calculated from $P$. The coordinates from $\mathrm{A}_{1}$ to $\mathrm{A}_{6}$ in $\Sigma_{a}$ are ${ }^{a} A_{i}=\left[\begin{array}{lll}{ }^{a} A_{i x} & { }^{a} A_{i y} & { }^{a} A_{i z}\end{array}\right]{ }^{T} \cdot{ }^{b} B_{i}$ and ${ }^{a} A_{i}$ are predefined constants. The lengths of the strain sensors are represented as $L_{s i}$. $L_{i}$ is obtained by the following equation.

$$
L_{i}={ }^{a} B_{i}-{ }^{a} A_{i}=\left[\begin{array}{lll}
L_{i x} & L_{i y} & L_{i z}
\end{array}\right]^{T} .
$$

The equation expresses the relation between $L_{i}$ and $P$. If we consider the difference between $L_{s i}$ and $L_{i}$ as the length $\dot{L}_{i}$ changes

$$
\dot{L}_{i}=L_{s i}-L_{i} \text {, }
$$

the following equation can be derived using the Jacobian determinant $J$.

$$
\dot{P}=J^{-1} \dot{L}_{i}
$$

From the difference between $L_{i}$ and $L_{s i}$, the change in position and rotation of the upper bar $\dot{P}$ is determined iteratively by Newton's method. The position and rotational

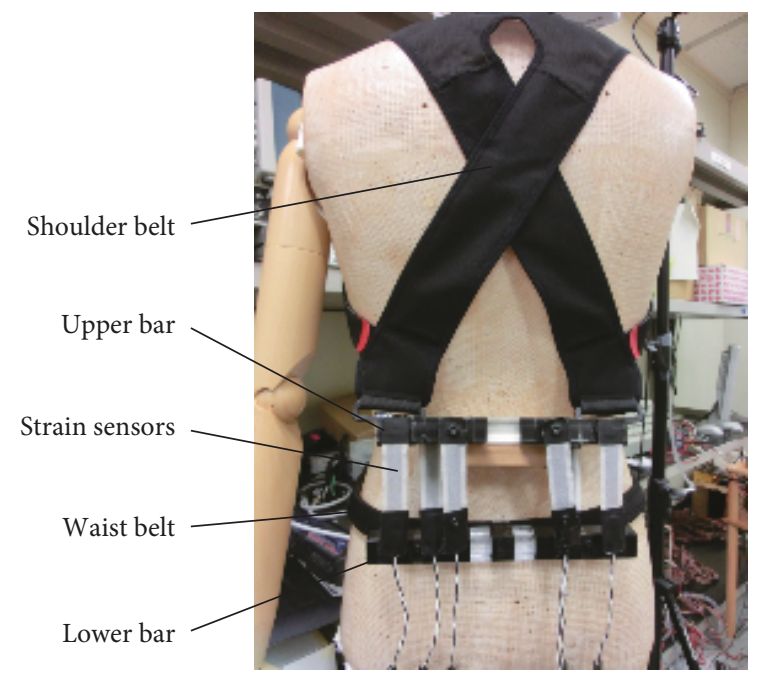

Figure 4: Prototype of LMMD on the low back of mannequin.

orientation of the upper bar $P$ is calculated by adding $\dot{P}$ to the previous $P$.

The prototype parallel-sensor mechanism we constructed is pictured in Figure 3. The parallel-sensor mechanism is built from two rectangular bars and six strain sensors, as shown in Figure 3. The dimensions of the upper and lower rectangular bars are $20 \times 200 \times 20 \mathrm{~mm}$ and $20 \times 260 \times 20 \mathrm{~mm}$, respectively. One terminal of each strain sensor is fixed on the upper bar, and the other terminal is fixed on the lower bar. Although the length between the two bars is about $100 \mathrm{~mm}$ in this picture, the default length is $80 \mathrm{~mm}$. The prototype weighs $235 \mathrm{~g}$.

The upper and lower bars are worn on the back over the first lumbar vertebra and the fifth lumbar vertebra with shoulder straps and a waist belt, as pictured in Figure 4 . The overall weight of the sensor mechanism with straps is 
$480 \mathrm{~g}$. The weight of the LMMD is lighter than that of the lumbar-motion monitor in [20]. This configuration assumes that the relative position and twist of the upper and lower bars change along with the motions of the first and fifth lumbar spines. As the relative position of the two bars changes, the capacitances of the strain sensors change. A capacitance-to-voltage converter processes the signals into voltages that are recorded by an analog-to-digital converter running on a desktop computer. The computer translates voltages into lengths of the strain sensors and iteratively calculates the relative position and rotation of the upper bar.

\section{Results and Discussion}

3.1. Parallel-Sensor Mechanism. First, a preliminary experiment was conducted to confirm the accuracy of the parallel-sensor mechanism. The lower bar was fixed on a table. The bend angle and rotation of the upper bar were manipulated by a hand. The computer sampled the lengths of the strain sensors at $20 \mathrm{~Hz}$ and calculated the displacement and rotation in three dimensions. An optical motion-capture system (Optitrack, NaturalPoint, USA) simultaneously measured the displacement and rotation of the upper bar from markers attached to the bar, as pictured in Figure 3. The motion-capture data were used as reference data. Overall, ten trials were performed to compare data from the parallelsensor mechanism and motion-capture system. Each trial lasted for about $25 \mathrm{~s}$. Typical results from these tests are shown in Figure 5. We calculated the cross-correlation values between the motion-capture data and the parallel-sensor mechanism data. The cross-correlation values of $x, y, z$, flexion-extension, side bending, and rotation were $0.84,0.93$, $0.83,0.84,0.94$, and 0.93 , respectively. The parallel-sensor mechanism data agreed well with those from the motioncapture system, with some error that we assume to be caused by errors in the individual length measurements.

The accuracy of the parallel-sensor mechanism was quantified using the residual errors between the parallelsensor mechanism and the motion-capture data. The mean and standard deviation (SD) of each dataset are listed in Table 1 . Table 1 also lists the ranges of motion (ROM). The means for side bending and rotation were within $2^{\circ}$ and were small. Compared to the data for these motions, the difference in the mean of the flexion-extension data was large. The means of the displacement data were nearly a function of the measured ROM. The ROM in the $x$-axis was limited by the maximum length of the sensors and was smaller than that of the other axes.

3.2. Lumbar-Motion Monitoring Device. We also tested LMMD with human subjects. Our study protocol complied with the Declaration of Helsinki. All participants were fully informed and provided written informed consent before participating in the study. The study and all procedures were approved by the Ethical Committee of Graduate School of System Informatics, Kobe University (Permission number: 28-03). The human subjects were ten volunteers of average dimensions: female: male $=1: 1$, mean age: $19.8 \pm 1.0$ years, height: $164.9 \pm 11.9 \mathrm{~cm}$, and weight: $58.1 \pm 18.2 \mathrm{~kg}$. The human subjects wore the LMMD and adjusted the tension of the straps to fit it to their low backs. Subjects were instructed to perform a motion typically encountered by nursing professionals, i.e., the transfer of a patient from bed to a wheelchair. Each subject performed the motion twice. The motion-capture system was used to obtain reference data as it was in the preliminary test. A selection of typical results is shown in Figure 6. The motion-capture data for flexion and extension had missing values due to a marker that was not detected by the motion-capture system. Excluding the missing data, the cross-correlation values of flexion-extension, side bending, and rotation were $0.78,0.89$, and 0.79 , respectively. Although the results differ somewhat, the LMMD data still agree reasonably with the reference data. Table 2 lists the mean and SD of the residual errors between the LMMD and the motion capture and the ROM of the LMMD. The mean for the side bending angle was relatively small, and flexionextension motions had more than double the error of the other motions.

3.3. Discussion. The comparison of data from the parallelsensor mechanism and motion-capture system demonstrates the validity of the proposed measurement device. The crosscorrelation values showed strong positive correlations. The difference in the means of the measurement and reference angles was less than $4.5^{\circ}$, which is less than $8.3 \%$ of the ROM of the device. These results show that the parallelsensor mechanism with strain sensors can measure rotation in three dimensions with reasonable accuracy.

In the tests with human subjects, the cross-correlation values also showed strong positive correlations. The ROMs of the side bending and rotation motion were small compared to that of flexion-extension and were less than $35^{\circ}$. The ROM of the flexion-extension was greater than $50^{\circ}$. Although these ROMs are expected for the single motion we tested, the device must measure rotation around all three axes to be effective. The differences in the means of the side bending and rotation measurements were less than $5^{\circ}$. In contrast, measurements of the flexion-extension motion returned errors greater than $10^{\circ}$. Two explanations of these errors seem likely. First, we note that arrangement of the sensors will lead to some error due to geometry. The Stewart platform has circular plates and places links at dispersed positions on those plates; however, the LMMD terminals are attached to sensors linked to a smaller range of positions on the upper and lower bars. In this arrangement, the sensor lengths change less as the upper bar moves than they would in the Stewart platform. The other cause is that the sensors are stretched along the curve of the low back so they do not remain straight as they move. The Jacobian matrix calculation then generates errors in the angle calculations; therefore, the length of the sensor is not accurately recorded as it bends. Improved bars and belts are required to prevent these errors. Sensors could be distributed evenly around the waist, and they could be kept straight. These limitations decrease the latitude of the design of bars and belts. For example, extremely downsizing the device is difficult. The errors yielded by this LMMD are acceptable for practical purposes, though, because the device is more wearable than similar 


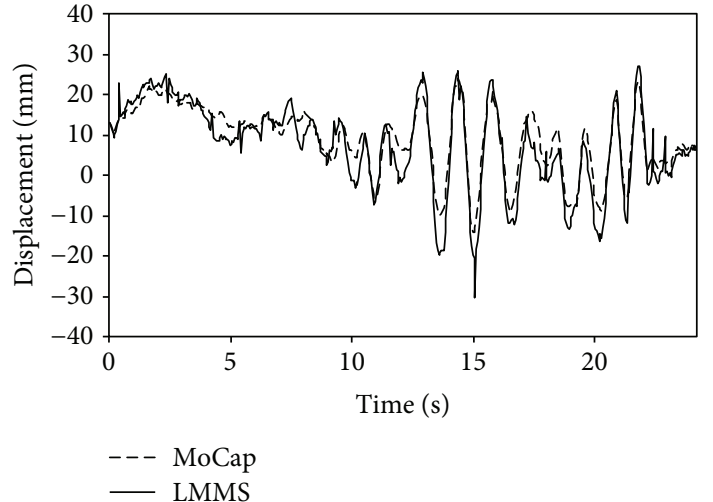

(a) $x$-axis displacement

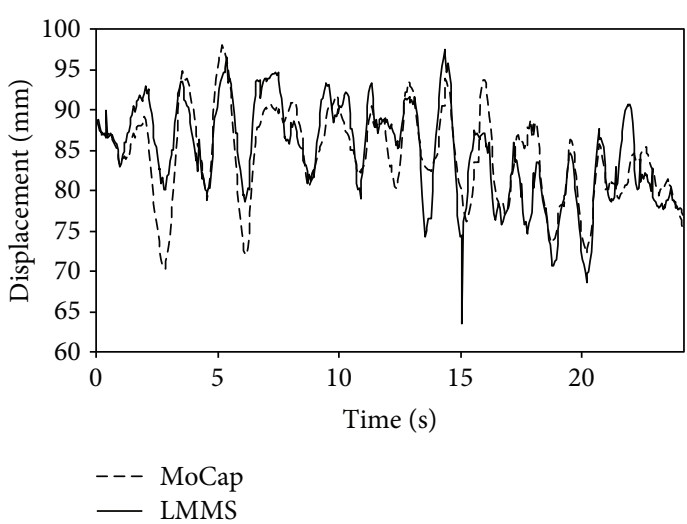

(c) $z$-axis displacement

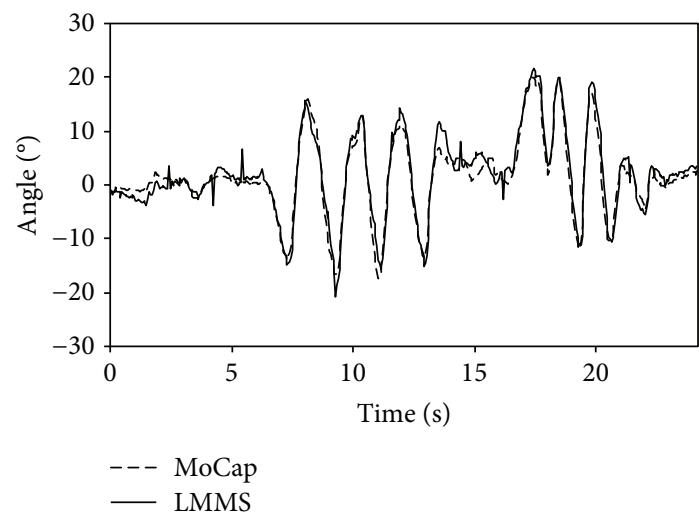

(e) Side bending

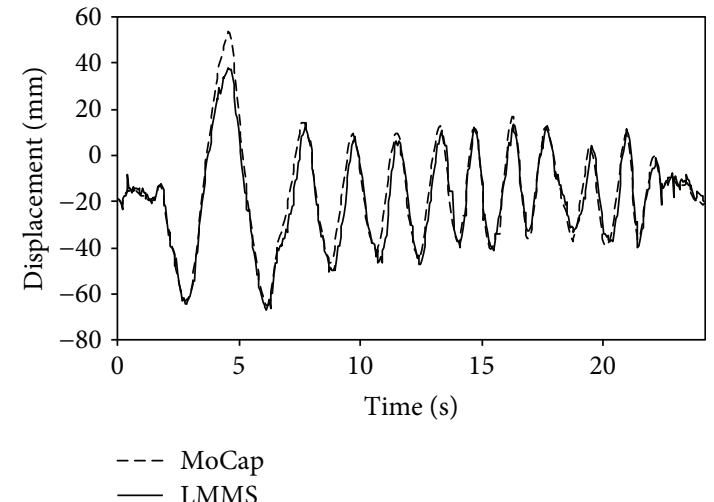

(b) $y$-axis displacement

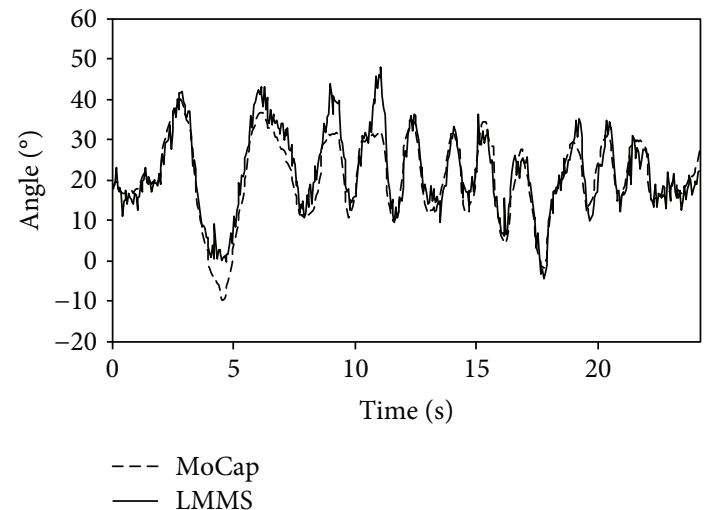

(d) Flexion-extension

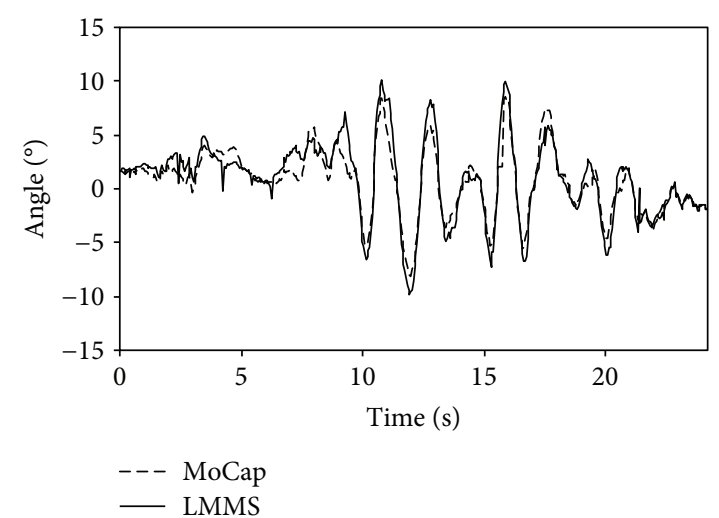

(f) Rotation

FIgURE 5: Typical time-series data for displacements and rotation angles when moving the parallel-sensor mechanism alone.

TABLE 1: Range of motion values and means and SDs of residual errors between measured and reference displacements and rotation angles in preliminary tests with the parallel-sensor mechanism on a bench.

\begin{tabular}{lcccccc}
\hline \multicolumn{4}{c}{ Displacement $(\mathrm{mm})$} & \multicolumn{3}{c}{ Angle $\left(^{\circ}\right)$} \\
Axis & $x$ & $y$ & $z$ & $\begin{array}{c}\text { Flexion- } \\
\text { extension }\end{array}$ & $\begin{array}{c}\text { Side } \\
\text { bending }\end{array}$ & Rotation \\
\hline ROM & 56.69 & 131.97 & 84.56 & 51.24 & 22.13 & 67.35 \\
Mean & 3.23 & 5.42 & 2.79 & 4.22 & 1.04 & 1.52 \\
SD & 3.24 & 5.43 & 2.36 & 3.43 & 1.02 & 2.17 \\
\hline
\end{tabular}

devices that have been proposed. The device could be effective for rough measurements and monitoring of multiaxis lumbar motion such as the transfer of a patient.

\section{Conclusion}

We developed a lumbar-motion monitoring device that uses flexible and stretchable strain sensors. The LMMD measured three-axis lumbar motion using a parallel-sensor mechanism with six strain sensors. The accuracy of the parallel-sensor mechanism was evaluated as the residual errors within $4.5^{\circ}$, and this may be sufficient for a wearable lumbar-motion 


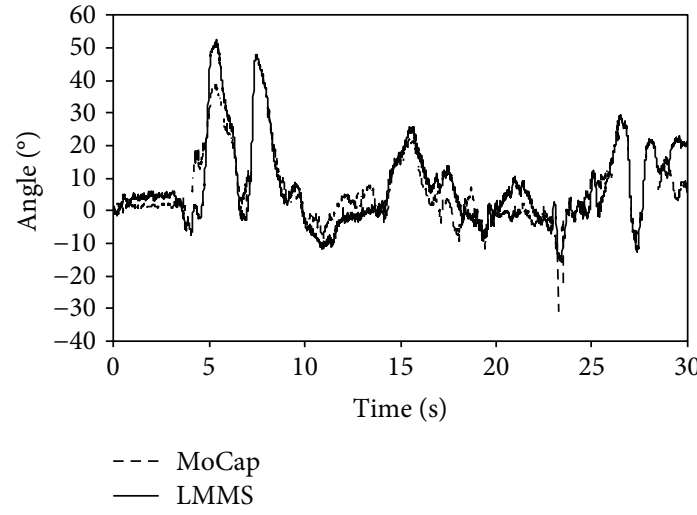

(a) Flexion-extension

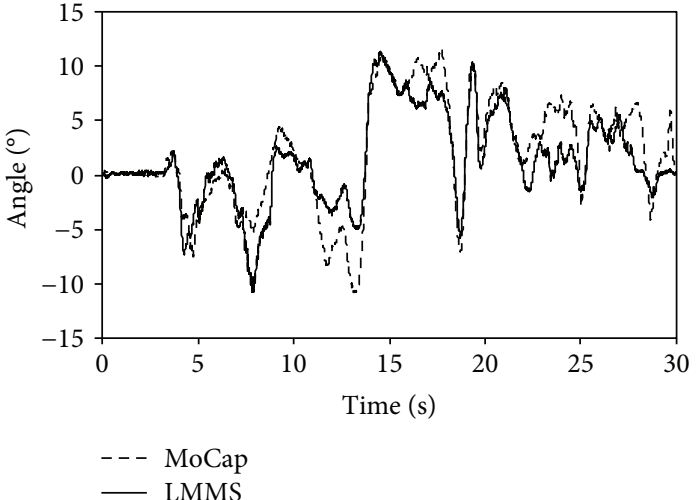

(b) Side bending

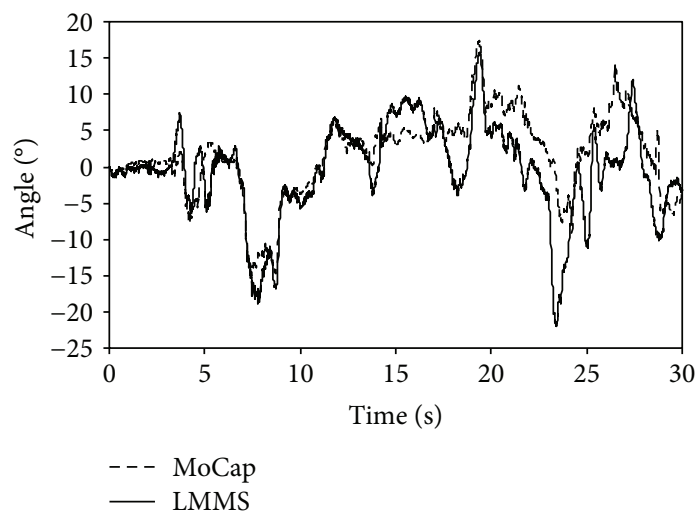

(c) Rotation

Figure 6: Typical time-series data of rotation angles for the device worn by human subjects.

TABLE 2: ROM values and means and SDs of residual errors between measured and reference rotation angles in human-subject experiments.

\begin{tabular}{lccc}
\hline Axis & Flexion-extension & Side bending & Rotation \\
\hline ROM $^{\circ}$ & 54.64 & 31.78 & 34.01 \\
Mean $^{\circ}$ & 11.11 & 3.26 & 4.64 \\
$\mathrm{SD}^{\circ}$ & 7.47 & 3.09 & 3.79 \\
\hline
\end{tabular}

monitoring device. The accuracy of the LMMD for tracking low-back movements of human subjects was evaluated against reference data from a motion-capture system. The accuracy of the LMMD was slightly inferior when worn by human subjects.

In future work, we plan to implement an arc-shaped bar that fits the user's waist better. If the sensors are evenly distributed to the arc-shaped bar, the LMMD could have high accuracy. We also plan to improve the device as a wireless device and to adapt the measurement device for continuous monitoring to make it practical for preventing LBP in the workplace.

\section{Data Availability}

The relevalent data used to support the findings of this study are included within the article.

\section{Ethical Approval}

All procedures used in this research were approved by the Ethical Committee of Graduate School of System Informatics, Kobe University (Permission number: 28-03).

\section{Disclosure}

The funders provided part of the experimental materials used for data collection, but this does not alter our adherence to the publication ethics of the journal on sharing data and materials.

\section{Conflicts of Interest}

YB and HO are employees of Bando Chemical Industries, Ltd. All other authors declare that they have no conflicts of interest.

\section{Acknowledgments}

This project was funded by a grant from Bando Chemical Industries, Ltd.

\section{References}

[1] C. Leboeuf-Yde, N. Klougart, and T. Lauritzen, "How common is low back pain in the Nordic population? Data from a recent 
study on a middle-aged general Danish population and four surveys previously conducted in the Nordic countries," Spine, vol. 21, no. 13, pp. 1518-1525, 1996.

[2] R. A. Deyo and J. N. Weinstein, "Low back pain," The New England Journal of Medicine, vol. 344, no. 5, pp. 363-370, 2001.

[3] D. Hoy, C. Bain, G. Williams et al., "A systematic review of the global prevalence of low back pain," Arthritis and Rheumatism, vol. 64, no. 6, pp. 2028-2037, 2012.

[4] A. Leclerc, F. Tubach, M. F. Landre, and A. Ozguler, "Personal and occupational predictors of sciatica in the GAZEL cohort," Occupational Medicine, vol. 53, no. 6, pp. 384-391, 2003.

[5] L. Punnett, L. J. Fine, W. M. Keyserling, G. D. Herrin, and D. B. Chaffin, "Back disorders and nonneutral trunk postures of automobile assembly workers," Scandinavian Journal of Work, Environment and Health, vol. 17, no. 5, pp. 337-346, 1991.

[6] H. Miranda, E. Viikari-Juntura, L. Punnett, and H. Riihimäki, "Occupational loading, health behavior and sleep disturbance as predictors of low-back pain," Scandinavian Journal of Work, Environment and Health, vol. 34, no. 6, pp. 411-419, 2008.

[7] I. Niedhammer, F. Lert, and M. J. Marne, "Back pain and associated factors in French nurses," International Archives of Occupational and Environmental Health, vol. 66, no. 5, pp. 349-357, 1994.

[8] J. Smedley, P. Egger, C. Cooper, and D. Coggon, "Manual handling activities and risk of low back pain in nurses," Occupational and Environmental Medicine, vol. 52, no. 3, pp. 160-163, 1995.

[9] A. M. Trinkoff, J. A. Lipscomb, J. Geiger-Brown, C. L. Storr, and B. A. Brady, "Perceived physical demands and reported musculoskeletal problems in registered nurses," American Journal of Preventive Medicine, vol. 24, no. 3, pp. 270-275, 2003.

[10] T. Videman, A. Ojajärvi, H. Riihimäki, and J. D. G. Troup, "Low back pain among nurses: a follow-up beginning at entry to the nursing school," Spine, vol. 30, no. 20, pp. 2334-2341, 2005.

[11] R. Ono, S. Yamazaki, M. Takegami et al., "Patient-reported disability in the general Japanese population was associated with medical care visits for low back pain, regardless of pain intensity," Journal of Orthopaedic Science, vol. 20, no. 4, pp. 742-749, 2015.

[12] W. E. Hoogendoorn, P. M. Bongers, H. C. W. de Vet et al., "Flexion and rotation of the trunk and lifting at work are risk factors for low back pain: results of a prospective cohort study," Spine, vol. 25, no. 23, pp. 3087-3092, 2000.

[13] T. R. Waters, V. Putz-Anderson, A. Garg, and L. J. Fine, "Revised NIOSH equation for the design and evaluation of manual lifting tasks," Ergonomics, vol. 36, no. 7, pp. 749-776, 1993.

[14] R. Norman, R. Wells, P. Neumann et al., "A comparison of peak vs cumulative physical work exposure risk factors for the reporting of low back pain in the automotive industry," Clinical Biomechanics, vol. 13, no. 8, pp. 561-573, 1998.

[15] A. J. Lisi, C. W. O’Neill, D. P. Lindsey, R. Cooperstein, E. Cooperstein, and J. F. Zucherman, "Measurement of in vivo lumbar intervertebral disc pressure during spinal manipulation: a feasibility study," Journal of Applied Biomechanics, vol. 22, no. 3, pp. 234-239, 2006.
[16] H.-J. Wilke, P. Neef, M. Caimi, T. Hoogland, and L. E. Claes, "New in vivo measurements of pressures in the intervertebral disc in daily life," Spine, vol. 24, no. 8, pp. 755-762, 1999.

[17] A. L. Nachemson, "The lumbar spine an orthopaedic challenge," Spine, vol. 1, no. 1, pp. 59-71, 1976.

[18] L. Milea, "Detection and tele-replication of human hand motions by a robotic hand," American Journal of Aerospace Engineering, vol. 2, no. 4, p. 30, 2015.

[19] M. C. Schall Jr, N. B. Fethke, H. Chen, and F. Gerr, "A comparison of instrumentation methods to estimate thoracolumbar motion in field-based occupational studies," Applied Ergonomics, vol. 48, pp. 224-231, 2015.

[20] W. S. Marras, F. A. Fathallah, R. J. Miller, S. W. Davis, and G. A. Mirka, "Accuracy of a three-dimensional lumbar motion monitor for recording dynamic trunk motion characteristics," International Journal of Industrial Ergonomics, vol. 9, no. 1, pp. 75-87, 1992.

[21] W. S. Marras, W. G. Allread, D. L. Burr, and F. A. Fathallah, "Prospective validation of a low-back disorder risk model and assessment of ergonomic interventions associated with manual materials handling tasks," Ergonomics, vol. 43, no. 11, pp. 1866-1886, 2000.

[22] W. S. Marras, K. G. Davis, B. C. Kirking, and P. K. Bertsche, "A comprehensive analysis of low-back disorder risk and spinal loading during the transferring and repositioning of patients using different techniques," Ergonomics, vol. 42, no. 7, pp. 904-926, 1999.

[23] H. Nakamoto, H. Ootaka, M. Tada, I. Hirata, F. Kobayashi, and F. Kojima, "Stretchable strain sensor based on areal change of carbon nanotube electrode," IEEE Sensors Journal, vol. 15, no. 4, pp. 2212-2218, 2015.

[24] H. Nakamoto, H. Ootaka, M. Tada, I. Hirata, F. Kobayashi, and F. Kojima, "Stretchable strain sensor with anisotropy and application for joint angle measurement," IEEE Sensors Journal, vol. 16, no. 10, pp. 3572-3579, 2016.

[25] T. Yamaji, H. Nakamoto, H. Ootaka, I. Hirata, and F. Kobayashi, "Rapid prototyping human interfaces using stretchable strain sensor," Journal of Sensors, vol. 2017, Article ID 9893758, 9 pages, 2017.

[26] D. Stewart, "A platform with six degrees of freedom," Proceedings of the Institution of Mechanical Engineers, vol. 180, no. 1, pp. 371-386, 1965.

[27] R. C. Merkle, "A new family of six degrees of freedom positional devices," Nanotechnology, vol. 8, no. 2, pp. 47-52, 1997.

[28] Y. Kitano and K. Yokota, "Method for measuring position and posture of the shoulder skeleton using parallel links," Transactions of the Japan Society of Mechanical Engeneers, Series C, vol. 79, no. 802, pp. 2004-2012, 2013. 


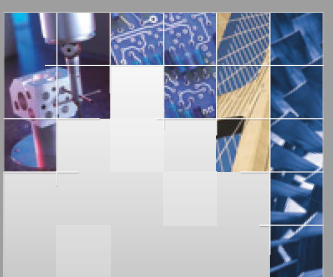

\section{Enfincering}
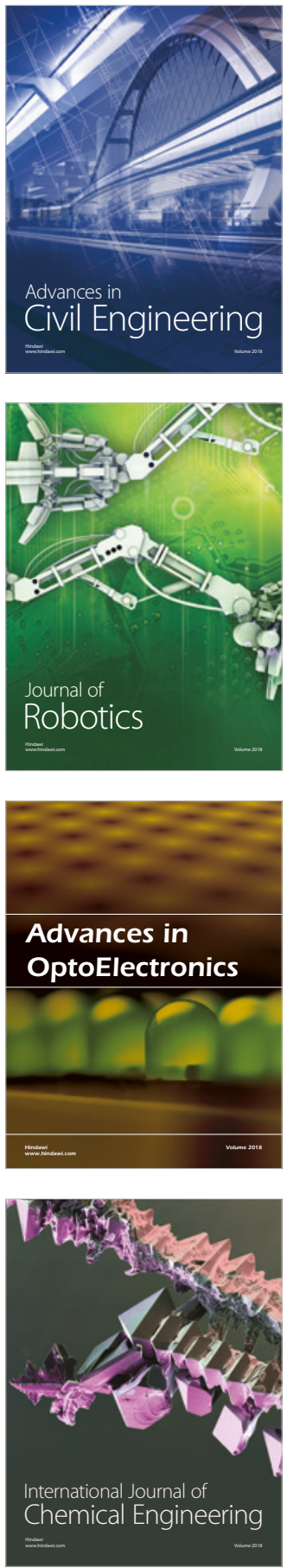

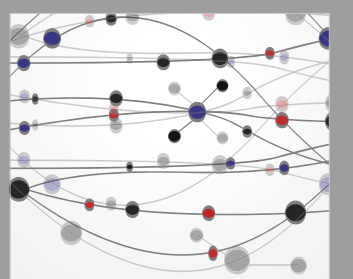

\section{Rotating \\ Machinery}

The Scientific World Journal

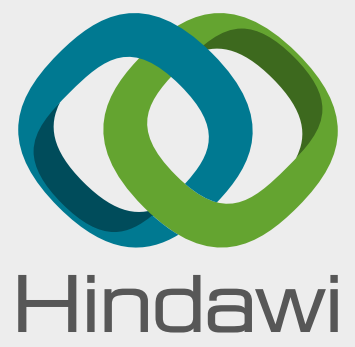

Submit your manuscripts at

www.hindawi.com
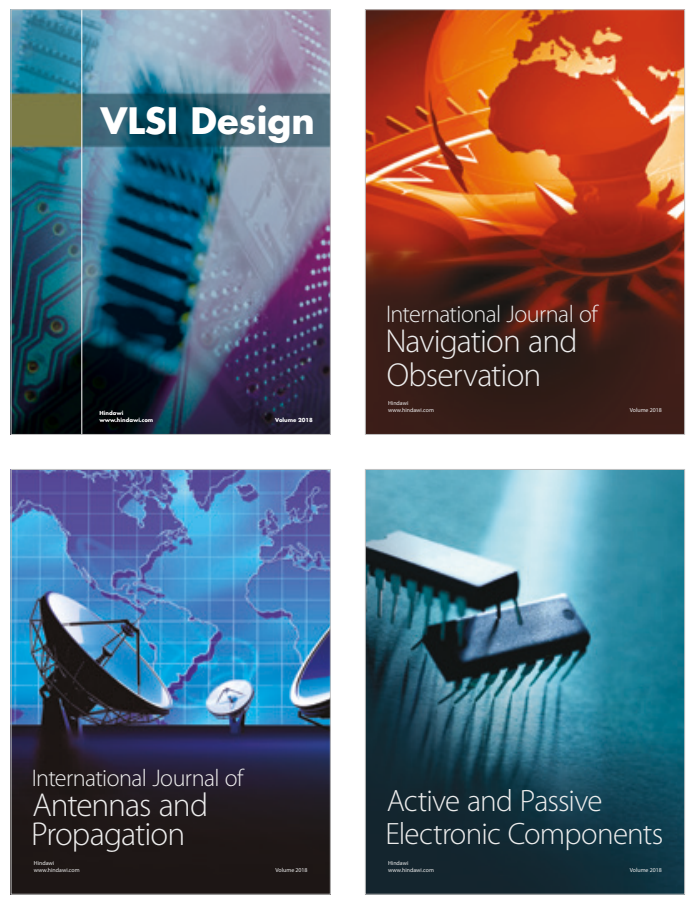
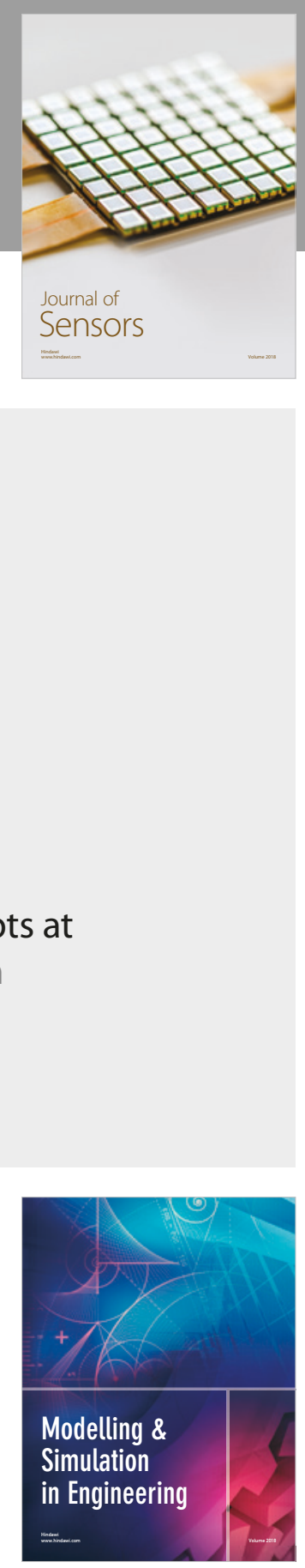

\section{Advances \\ Multimedia}
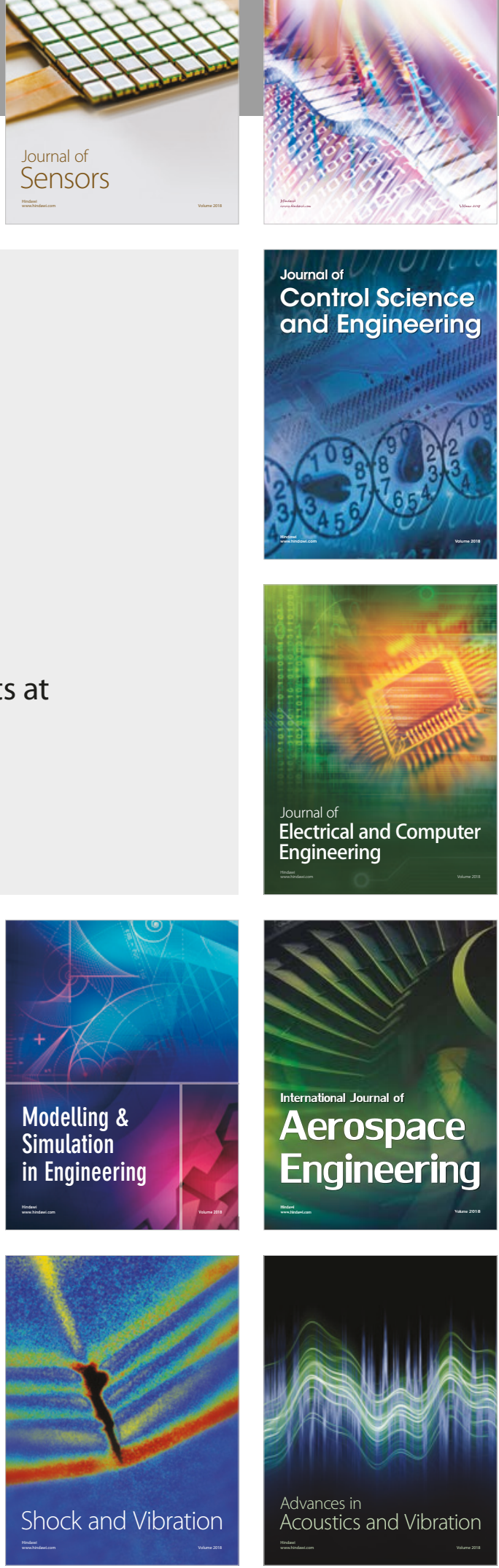